\title{
Secuencias didácticas en el campo †ridimensional con la interacción de medios manuales y digitales
}

\author{
Didactic sequences in the three- \\ dimensional field with the manual \\ and digital means interaction
}

María cabezas*

$<$ Resumen>

El conocimiento de los fenómenos morfo-espaciales, más allá de la intuición racionalizada, la mecánica operativa, criterios que se utilice para su conformación y las instancias de configuración geométrica y morfológica desde el campo de la educación visual, se ven favorecidas por una integración de medios manuales y digitales en su enseñanza y aprendizaje.

$<$ Abstract>

The knowledge of the morfo-space phenomena, beyond the rationalized intuition, the operative mechanics, approaches that it are used for its conformation and the instances of geometric and morphological configuration from the field of the visual education, are favored by an integration of manual and digital means in their teaching and learning.

<PALABRAS CLAVE >

FORMA / ESPAC 10 / ENSEÑANZA /

MEDIOS ANÁLOGO-DIGITALES

-KEY WORDS $>$

FORMS / SPACE / TEACHING / ANALOGICAL-DIGITAL

MEANS

\section{Introducción}

Este espacio de exploración y reflexión tiene como sustento la propuesta pedagógica y plan de trabajo que la autora desarrolla, como titular de la cátedra de Introducción a la Comunicación Visual, asignatura de $1^{\text {er }}$ año de la carrera de Arquitectura, de la Facultad de Arquitectura, Urbanismo y Diseño de la Universidad Nacional de Mar del Plata, Argentina.

Se plantean los fundamentos de la educación visual en tres ejes pedagógicos: el perceptivo, el geométrico y el morfológico. Estas áreas de conocimiento, que tienen autonomía teórica, subsidian los instrumentos conceptuales requeridos para la elaboración

\footnotetext{
Arq. María Cabezas. Prof. Titular de la cátedra Introducción a la Comunicación Visual, 1er año, carrera Arquitectura. Codirectora del Grupo de Investigación Geometría, abocado al estudio, capacitación y transferencia a la cencia de desarrollos específicos sobre la Nuevas Tecnolóís aplicadas a la Formación. Centro de Estudios de Diseño (CED), Facultad de Arquitectura, Urbanismo y Diseño (FAUD), Universidad Nacional de Mar del Plata (UNMDP), Argentina (mcabezas@mdp.edu.ar).
} 
de los procesos y productos en la práctica proyectual. Estos saberes de distinto carácter confluyen y se vinculan con un sabe instrumental de técnicas gráficas, que actúan como soporte visual y hacen visible la imagen.

La propuesta general se estructura con la interrelación de las diferentes unidades temáticas, constituyendo una secuencia didáctica integrada, con el sentido de construir el conocimiento con un grado de complejidad creciente y permitiendo articular los conceptos teóricos necesarios.

Considerando las evaluaciones realizadas en los últimos años respecto a los procesos de aprendizaje, resultados obtenidos y experiencias realizadas de integración del uso de medios analógicos y digitales en la educación visual, es objetivo compartir un recorte de los temas de estudio: La experimentación en el campo tridimensional y reflexionar sobre la integración de medios manuales y digitales en su aprendizaje.

Se presentarán los principios que se constituyen en instrumentos conceptuales capaces de generar forma tridimensional. Secuencias que introducen en la problemática de la forma y el espacio, en la configuración de sus límites y en sus relaciones estructurales. En paralelo, el uso de medios para el estudio y materialización de esas formas en el espacio, procesos analíticos, registro gráfico de ideas y exploraciones digitales.

La finalidad de este trabajo es una contribución orientada a la formación inicial en la carrera de Arquitectura, sobre una temática experimentada en sus aspectos teóricos y prácticos a lo largo de muchos años, y a su vez abierta a posibles modificaciones, al transcurso de nuevas experiencias.

\section{Магсо general}

Los estudiantes de $1^{\text {er }}$ año, poseen diferentes habilidades y experiencias educativas previas a su ingreso a la Universidad. Se les solicitará en poco tiempo, desde distintas asignaturas, que observe, analice, interprete, contextualice históricamente, que dibuje, lea, arme objetos que aparecen bajo una forma. Objetos que, partiendo de formas geométricas elementales avanzan hacia formas arquitectónicas.

Es nuestro compromiso como docentes asumir las distintas formaciones y capacitaciones, contribuir a que no se excluyan por ciertas diferencias, sino que se sientan parte de un proyecto colectivo: la enseñanza de la arquitectura.
El mundo físico de los objetos y cosas que nos rodean es percibido a través de una forma. Todo hecho urbano-arquitectónico se manifiesta ineludiblemente a través de un lenguaje de formas.

La problemática de la forma en la enseñanza de la arquitectura constituye un tema central y eje de diseño. Es un caso particular de la enseñanza universitaria como problema de conjunto, por lo que la afectan las específicas circunstancias de orden histórico, social, político, económico, ambiental, que definen nuestro tiempo presente, nuestra cultura de la fragmentación, de la inmediatez; haciendo suyos los fundamentos científicoepistemológicos y pedagógico-didácticos que atraviesan toda propuesta educativa.

El proyecto arquitectónico opera con los espacios y las formas, coherente por una relación directa entre el pensamiento y los instrumentos con que se los concretiza. Instrumentos cargados de intencionalidad, nunca neutrales.

Desde lo pedagógico, los principios que sustentan este enfoque centran la concepción del conocimiento como construcción social, capaz de promover el aprendizaje a través del diálogo, el intercambio, la divergencia de opiniones. La enseñanza y aprendizaje es entendida como procesos constructivos y de comunicación multidireccional.

La intervención didáctica conduce a rescatar los conocimientos y experiencias previas de los estudiantes en su contexto sociocultural, produciendo sucesivas aproximaciones a los nuevos objetos de conocimiento.

Se buscan estrategias cognitivas que ayuden al estudiante a una creciente autonomía y autorregulación de sus propios procesos de aprendizaje y transferencia. Se ha comprobado en diferentes experiencias realizadas que hay una eficaz compatibilidad pedagógica entre el tema específico a tratar del espacio y la forma y la enseñanza asistida por computadora.

Es la predisposición espontánea de los estudiantes a operar con los medios informáticos y su potencial poder de simulación, lo que fomenta la integración de medios como recurso pedagógico.

La incorporación de las nuevas tecnologías de la información y comunicación a la enseñanza, implica un desafío a la capacidad crítica y creativa del docente, no una sustitución de su rol. El uso de los medios informáticos tiene como componentes la capacitación y planificación, variables que juegan a favor de la calidad de la enseñanza y consecuentemente de los aprendizajes.

El uso de la computadora en la educación, ha permitido ganar tiempos reales y simbólicos para el aprendizaje significativo y socialmente compartido, llegar a la realidad del contexto y del sujeto en sus complejas y múltiples interacciones, favoreciendo la apropiación crítica de todos los productos de la cultura y liberando los espacios institucionales de la carga impuesta por el uso exclusivo de los recursos tradicionales.

\section{Estudios previos al campo tridimensional}

La experiencia en el campo tridimensional constituye la tercera unidad pedagógica, precedida por:

\section{Unidad 1: Análisis visual / Croquis}

Serie de ejercicios que desarrollan la capacidad de ver, el paso del mirar al ver consciente. Proveen la apertura hacia la captación de parámetros del mundo visual: forma, luz y sombra, textura, color, etc., y de la intencionalidad: percepción, memoria, imaginación, intuición. Trabajos con variables gráficas que otorgan sentido al punto y la línea como componentes básicos de la alfabetidad visual.

Se trabaja con diferentes herramientas y soportes generando grafías. Con escalas de valores como gradaciones tonales.

Se desarrolla una metodología del dibujo que abarca la observación-comprensiónrepresentación. El croquis como dibujo de análisis y de síntesis.

\section{Unidad 2: Experimentación \\ en el campo bidimensional / Percepción en el plano}

Etapa orientada a la comprensión del concepto de estructura formal y la lógica geométrica subyacente.

Se trabaja con las figuras básicas: triángulo, cuadrado y círculo; con su estructura portadora, estructura modular, líneas internas, relaciones y posibles transformaciones.

Se reproducen mallas planas regulares que se utilizan como soporte en el planteo de diferentes organizaciones y propuestas de 
distintas lecturas por variables de tratamientos gráficos. Se trabaja en la propuesta de motivos que permiten con diferentes movimientos en e plano generar bandas planas y mosaicos.

Se inicia la práctica instrumental con herramientas de dibujo de precisión.

\section{Secuencias didácticas y desarrollo de experiencias en el campo tridimensional}

El sistema didáctico está compuesto por los docentes de la cátedra, los estudiantes y los fenómenos morfoespaciales como objeto de conocimiento.

Atendiendo a las particularidades que impone dicho objeto y al perfil de los estudiantes es que se opta por un camino metodológico que va de lo general a lo particular, pues se trata de un aprendizaje fuertemente ligado a lo perceptivo.

El estudio de las formas, desde la perspectiva del diseño es el estudio de las formas espaciales y perceptibles por el hombre.

La secuencia en este módulo de trabajo es:

\section{Fase 1: Generación \\ de superficies}

El objetivo es introducirse en el mundo tridimensional, con una sola consigna: generar una superficie.

El trabajo es en equipos de tres estudiantes, lo que permite en una primera etapa incentivar la conciencia grupal y socia del aprendizaje.

La construcción es en maqueta, utilizando diversos materiales: hilos, varillas de madera, alambre, elástico, etc. De manera intuitiva, sin ningún condicionamiento, con un alto grado de libertad, los estudiantes dejan fluir su creatividad e imaginación. Aparecen así materializados y visibles, los elementos geométricos capaces de generar forma. Se descubre la línea como unidad de generación, rectas y curvas con diferentes comportamientos en el espacio, generan en sus movimientos distintas superficies.

Luego de esta primera experiencia, viene teoría. Ahora las superficies tienen elementos que las generan, tienen leyes que las dirigen, tienen nombres que las designan.

\section{Fase 2: Estructura formal. módulo didáctico: cubo}

Nuestro objetivo es el conocimiento de las estructuras formales, por lo que utilizaremos consideraciones geométricas como medio para llegar a ellas.

En toda forma podemos referirnos a dos estructuras. Una estructura abstracta definida por el conjunto de elementos que definen sus propiedades fundamentales: líneas, planos, ejes, que son elementos conceptuales. Esta estructura es la que determina la forma, es la que hace posible la operación mental y consciente de concebirla y analizarla.

Y una estructuración concreta referida al modo en que una forma es constituida, a su existencia real, posibilitad de percepción inmediata, realización específica, a su construcción.

Las estructuras abstractas y concretas están vinculadas. La intencionalidad actúa sobre la estructuración concreta como factor capaz de poner en evidencia y valorizar selectivamente algunos de los elementos de la estructuración abstracta.

Cada forma posee una estructura propia, más o menos visible, manifiesta y coherente que podríamos llamar estructura portadora de esa forma. Es definida por líneas organizadas de determinada manera que se cruzan en determinados puntos, capaces de permiti reconocer la forma que soportan. Son puntos y líneas de máxima caracterización de esa forma.

Para comprender la estructura portadora del cubo, partiremos de la figura dibimensional cuadrado. Cada una de las líneas que estructuran esta figura son ejes de simetría bilateral de la misma. Esto significa que particionan la figura en partes iguales. $\mathrm{O}$ a la inversa, que la figura queda construida por duplicación con relación a cada eje.

Estas estructuras analizadas en las figuras básicas planas, las encontramos análogamente por extensión en las formas tridimensionales correspondientes. Un punto que se desplaza genera una línea, una línea que se desplaza genera un plano, un plano que se desplaza genera un sólido. Podemos generar un cubo a partir de un cuadrado que se desplaza sobre un eje.

Comienza el análisis y lectura entonces a partir de la traslación de los ejes estructurantes del cuadrado, que en su traslación generan planos. Cada eje de simetría del cuadrado, genera un plano de simetría del cubo. Se puede verificar para todas las direcciones espaciales. La intersección de éstos planos, determina ejes de simetría, sus intersecciones el centro de simetría del cubo. En este juego de ida y vuelta que nos permite la geometría con el potencial criterio de reversibilidad, determinamos la estructura abstracta del cubo.

Podemos sintetizar los planos de simetría del cubo en dos familias: Ios planos diagonales y los planos paralelos a las caras, con sus variables posicionales en el espacio. $Y$ esquematizar en tres sistemas los ejes de simetría del cubo: los que pasan por los centros de las caras opuestas, por los puntos medios de las caras opuestas y por los vértices opuestos.

En todos los casos, las intersecciones de los ejes de simetría, geométricamente un punto, es el único centro de simetría de cubo. De manera análoga podemos analizar la estructura, planos, ejes y centro de simetría de otros cuerpos regulares [Figuras 1 y 2].

Experimentación: La consigna básica es trabajar con los planos de simetría de sólidos regulares: cubo, tetraedro, octaedro. El objetivo, lograr a través de determinadas operaciones geométricas de división, adición y sustracción, particionar en porciones guales entre sí, estas formas culturalmente reconocidas.

La riqueza de esta ejercitación, es que la exploración se lleva a cabo con toda la forma, no sólo sobre su envolvente, lo que permite investigar en las líneas espaciales y planos internos de cada forma.

Esta serie introduce en el estudiante una conciencia espacial y estructural tridimensional que permite la visualización interna de las aparentes formas sólidas. Resulta favorable para el desarrollo de la inventiva, que el estudiante se ubique como productor de los fenómenos morfoespaciales y no como repetidor de nociones.

Se usa la maqueta analógica como medio para el estudio y la materialización de las formas y el espacio, considerado como el medio más eficaz para hacer visible los elementos y sus relaciones.

El trabajo grupal incentiva el diálogo e intercambio de ideas, desarrolla una actitud reflexiva y una conducta solidaria [Figura 3]. 
1

三网三用

$-1 ! 1+4$

$\equiv-v \equiv$

Hi
2

planos de simetria paralelos a las caras
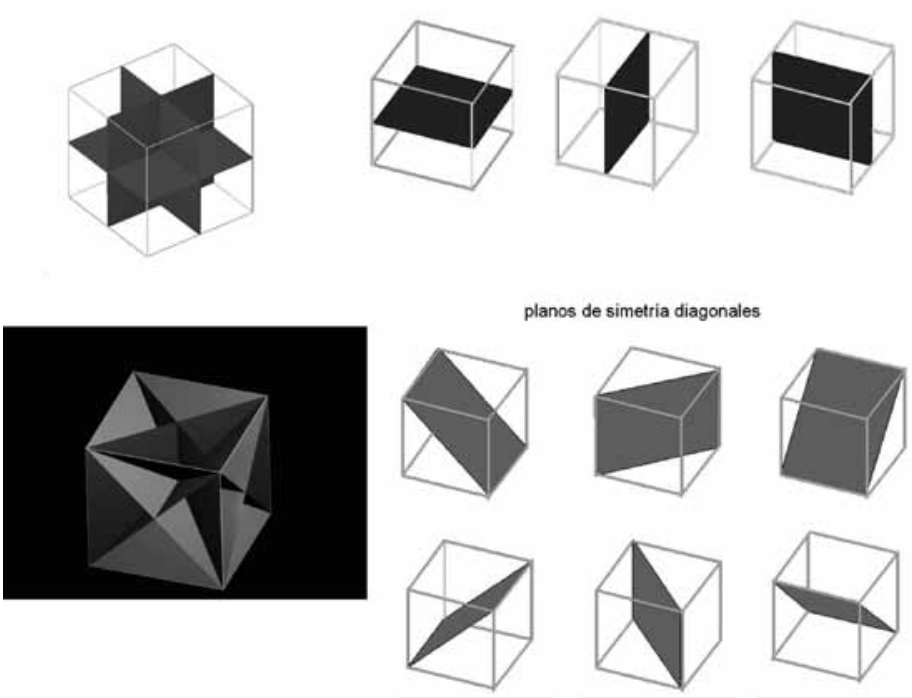

3
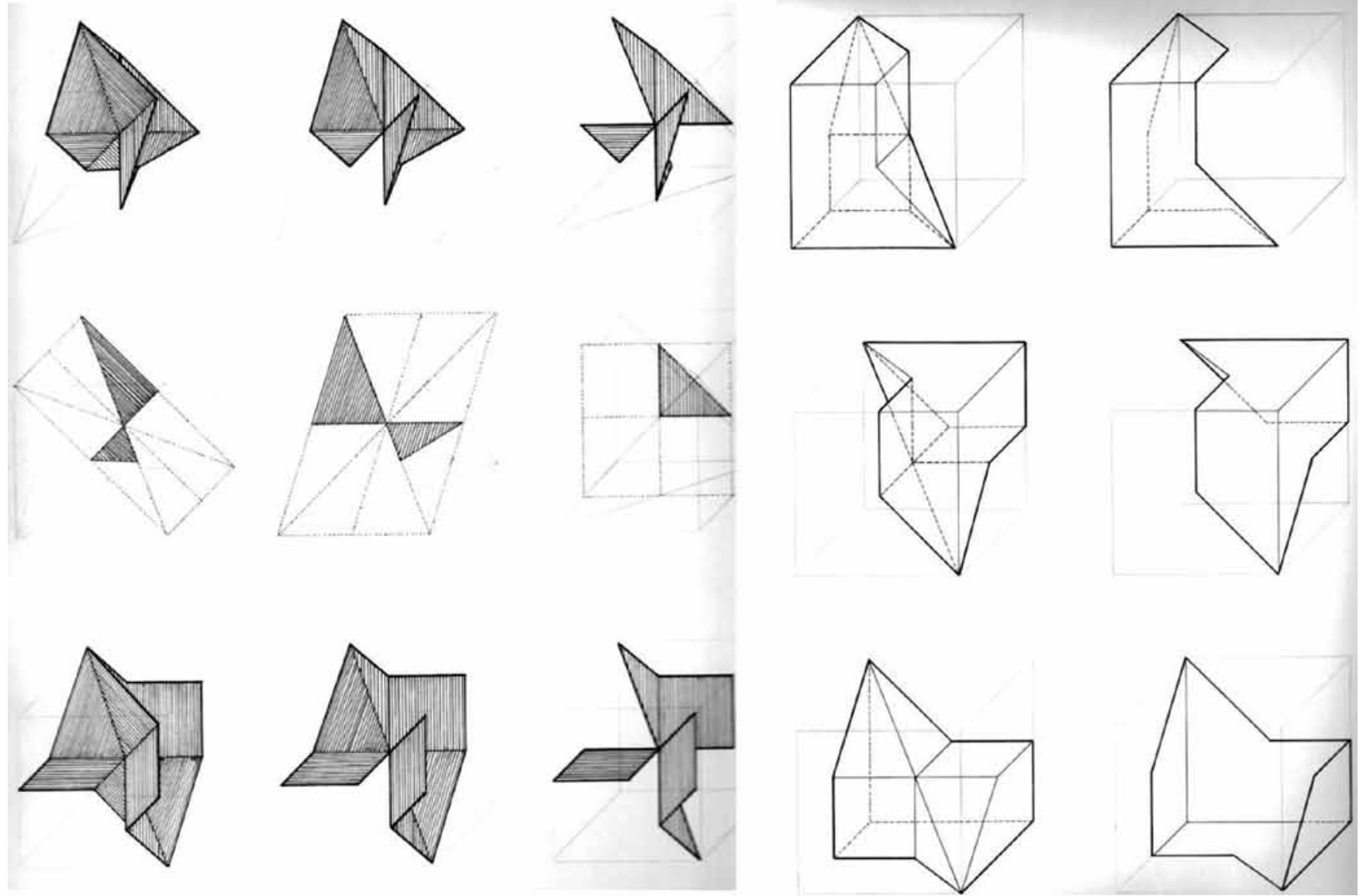


\section{Fase 3: Diferentes lecturas de la propuesta}

Cuando hablamos de lecturas o significación nos referimos a cierta disposición espacial de los elementos, a la interpretación y calificación de los mismos y de sus propiedades.

Entidades definidas unívocamente desde la geometría, no lo resultan desde la morfología en que las oposiciones básicas de los elementos que constituyen la forma inducen a muy diferentes lecturas.

La graficación se constituye en un potencial instrumento que aumenta las posibilidades de percepción y significación de las formas, atendiendo y respondiendo a determinadas intencionalidades. Así incorporamos a la generación de formas desde una misma estructura abstracta, cualidades y calidades en sus elementos que van modificando sus lecturas definiendo diferentes estructuras concretas.

Estos principios de relectura a través de dibujo, necesita el reconocimiento de todos los rasgos, propiedades y atributos del objeto a representar. Aparece toda la problemática del dibujo con la carga intencional y activa que este tiene al momento de comunicar [Figura 4].
4
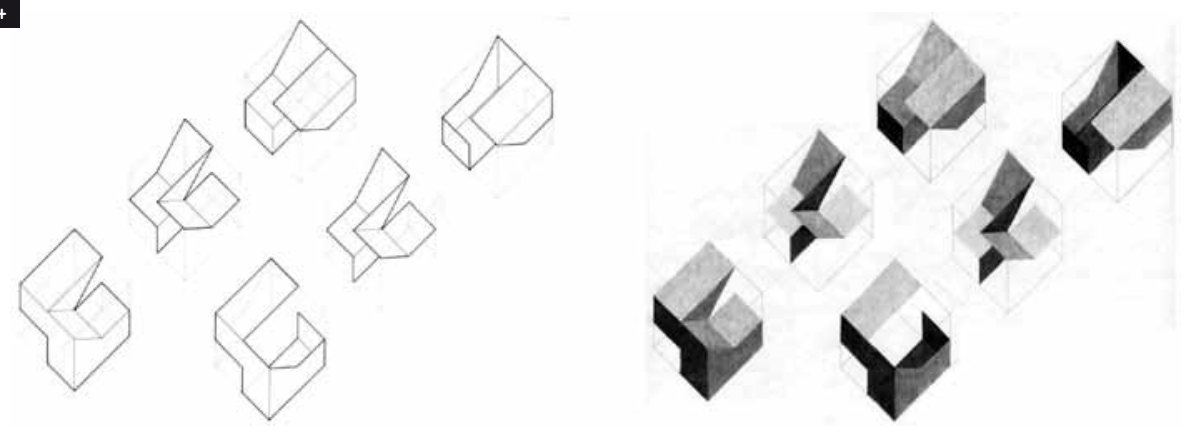

5
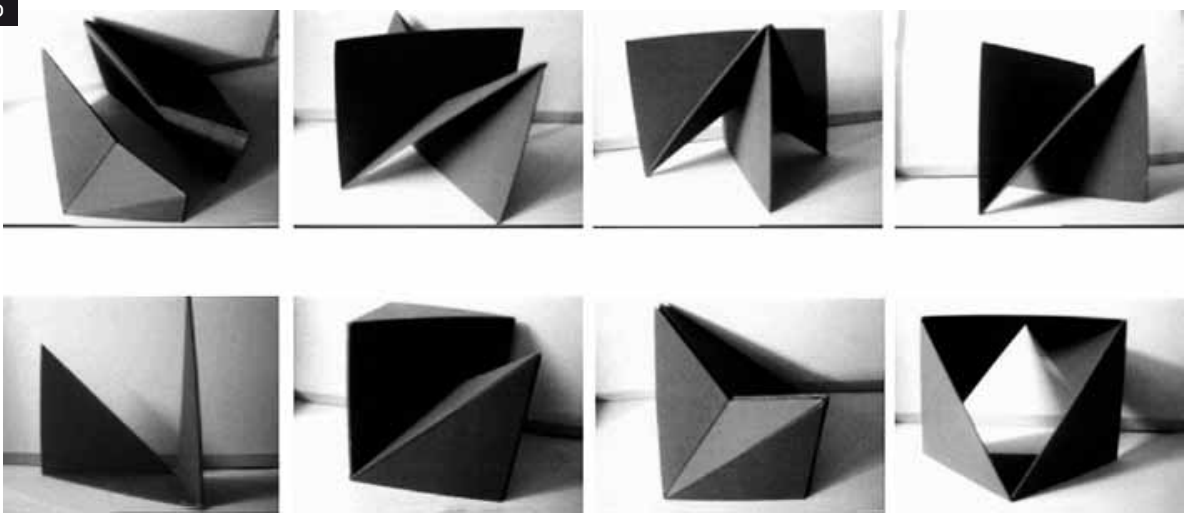

Figura 1: Planos de simetría en el cubo; dibujos de la autora, material de clases teóricas.

Figura 2: Ejes de simetría en el cubo; dibujos de la autora, material de clases teóricas.

Figura 3: Operaciones de división, adición y sustracción en los procesos formales; registros gráficos de propuestas de estudiantes del taller de Introducción a la Comunicación Visual

Figura 4: Lecturas de espacio, envolvente y sólido; estudiantes del taller de Introducción a la Comunicación Visual.

Figura 5: Selección de diferentes imágenes locales, luego de un recorrido; estudiantes de taller de Introducción a la Comunicación Visual.
Experimentación: Tiene el sentido de transformar la segmentación de partes iguales entre sí, en base a los modelos realizados en las maquetas de estudio de la primera etapa, en lecturas diferentes, que serán materializadas en nuevas maquetas.

Se reproducen nuevos modelos reconstruyendo como variantes, una primera lectura como espacios dentro de la forma virtual base con la que se trabajó, materializada por las partes de planos de simetría producto de las operaciones realizadas. Una segunda lectura se instaura materializando la forma por sus caras, con la impronta que dejan en éstas las secciones de los planos utilizados, tomando protagonismo la envolvente superficial. Al materializar cada parte como un componente sólido, se establece una tercera lectura, de volumen.

\section{Fase 4: Relevamiento con la videocámara digital}

La relación hombre-objeto establecida durante la construcción de la maqueta analógica, se modifica al variar los puntos de vista posibles ahora registrados por la cámara digital. Aparece una nueva noción de escala y consecuentemente se van descubriendo nuevas imágenes de las formas locales [Figura 5].

Experimentación: Se realiza un relevamiento de las propuestas morfoespaciales mediante el uso de la videocámara digital. Se constituye esta etapa en un innovador y valioso aporte al aprendizaje de esta específica temática.

Esta etapa de investigación por parte de los estudiantes, permite transitar nuevos caminos hacia la toma de conciencia de los fenómenos tridimensionales y otras lecturas de los rasgos más significativos en cada propuesta. Los resultados que se dan en el campo geométrico intuitivo del que parten son validados en el campo perceptivo.

\section{Fase 5: Comunicación gráfica}

Los procesos analíticos y propuestas formales, fueron registrados a mano alzada utilizando la potencialidad del croquis como registro gráfico de ideas.

En esta etapa se desarrollan las perspectivas paralelas como dibujo analítico constructivo, que mantiene la unidad de la imagen, instrumentos adecuados para el reconocimiento y análisis formal y espacial. El uso de axonometrías con operaciones de despiece y explotadas completan la representación de las propuestas. 
Algunos estudiantes que manejan os sistemas CAD, elaboran maquetas electrónicas, aportando al taller nuevas visualizaciones y recorridos en tres dimensiones [Figuras 6 y 7]

\section{Fase 6: Organizaciones formales}

Por repetición de las unidades obtenidas en la fase anterior, se definen organizaciones geométricas: central, radial, lineal, agrupada y en trama

Las relaciones estructurales entre las partes y el todo definen conceptos como: subordinación de elementos, llenos o vacíos, físicos o virtuales, núcleos centrífugos o centrípetos, características de cerrado, abierto, dinámico, estático; aperturas al crecimiento o formas rematadas, marcación
6

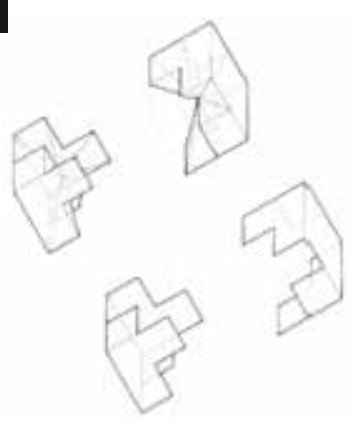

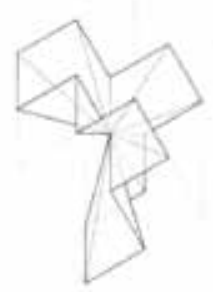
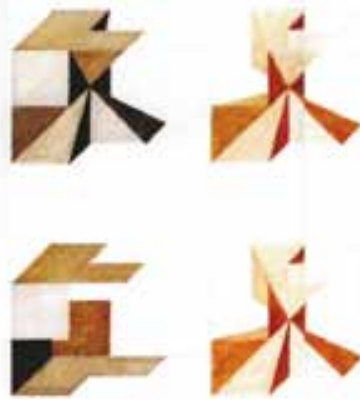
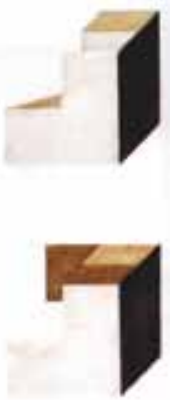

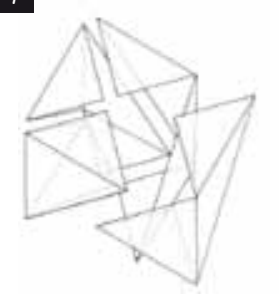

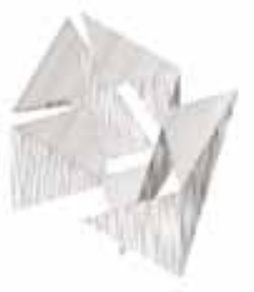

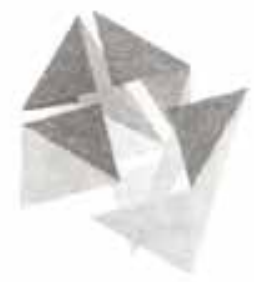

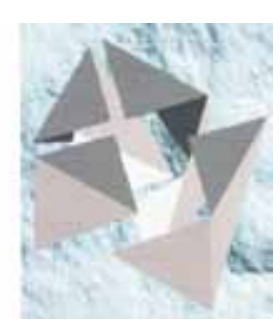

8
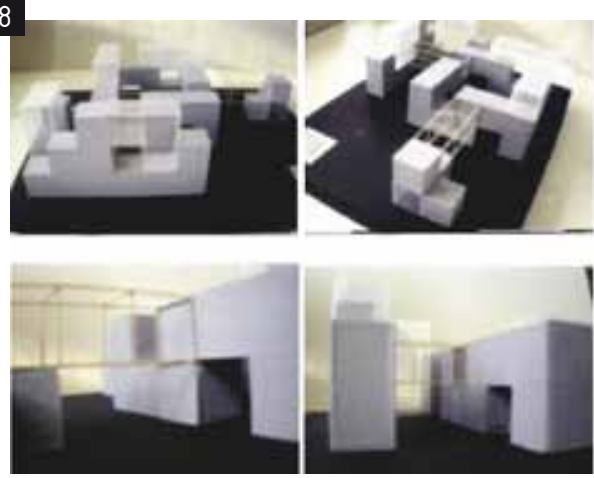
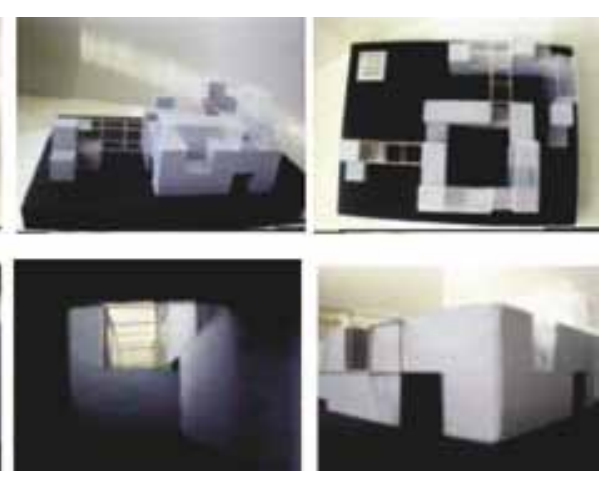

Figura 6: Representación en perspectiva paralela, con valor de línea y valor de plano: estudiantes del taller de Introducción a la Comunicación Visual.

Figura 7: Representación de la envolvente en diferentes técnicas gráficas; estudiantes del taller de Introducción a la Comunicación Visual.

Figura 8: Recorrido con la cámara digital de la propuesta de organización formal; estudiantes del taller de Introducción a la Comunicación Visual.

de direcciones espaciales y ritmos por disposiciones de elementos a intervalos perceptibles; agrupaciones por proximidad o por propiedades formales dominantes [Figura 8].

Experimentación: las propuestas de organizaciones geométricas se construyen en maquetas analógicas, de estudio y luego de presentación, trabajando los opuestos: material-virtual, opaco-transparente. También se explora con medios digitales haciendo recorridos con la videocámara.

Esta ejercitación se utiliza para la práctica de Geometría Descriptiva, como sistema de dibujo descompositivo capaz de mostrar los principios geométricos y los aspectos dimensionales en la representación de plantas, cortes y vistas. Se conceptualizan los trazados de sombra en axonometrías y en geometral, como sistemas capaces de enfatizar las estructuras formales y la creación o destrucción del espacio.

\section{Reflexiones}

Las sucesivas experiencias a la implementación de la enseñanza de los fenómenos morfoespaciales dirigido a estudiantes de $1^{\text {er }}$ año de arquitectura desde el campo de la educación visual, nos permite evaluar la eficacia de la modalidad implementada en el trabajo de taller.

Cabe mencionar la buena predisposición del estudiantado en el desarrollo de toda la temática planteada. Esto tiene relación con la manera de entender la enseñanza, con una metodología de experimentación basada en situaciones lúdicas, que es una de las formas más naturales inherentes al ser humano para la integración de habilidades y construcción del conocimiento.

La combinación de medios digitales con los sistemas tradicionales, proponen nuevas alternativas para la formación, en la dirección de lograr una mayor autonomía y flexibilidad de los procesos de enseñanza y aprendizaje.

El nuevo espacio de diálogo entre los modos de producción manual y digital, el ir de un medio a otro, aumenta los niveles de creatividad, favorece una mayor actitud exploratoria e incentiva al estudiante al comenzar a transitar un camino hacia otras y nuevas formas de pensamiento, en una nueva configuración enseñanza y aprendizaje. 